Original Paper http://ajol.info/index.php/ijbcs http://indexmedicus.afro.who.int

\title{
Hydrochimie et potabilité des eaux du bassin versant du Nchi dans le plateau Bamoun (Ouest Cameroun)
}

\author{
Z. MFONKA ${ }^{1 *}$, J. R. NDAM NGOUPAYOU $^{1}$, P. D. NDJIGUI ${ }^{1}$, M. ZAMMOURI ${ }^{2}$, \\ A. KPOUMIE $^{3}$ et E. RASOLOMANANA ${ }^{4}$ \\ ${ }^{I}$ Université de Yaoundé I, Faculté des Sciences, BP 812 Yaoundé, Cameroun. \\ ${ }^{2}$ Université de Tunis El Manar Faculté des Sciences, Tunisie. \\ ${ }^{3}$ Université de Maroua, Faculté des Sciences, Cameroun. \\ ${ }^{4}$ Université d'Antananarivo, Institut et Observatoire de Géophysique Appliquée \\ Antananarivo, Madagascar. \\ *Auteur correspondant, E-mail : zakarimfonka@yahoo.fr, Tél : (237)674753688/261332015759
}

\section{RESUME}

Comme dans la plupart des métropoles camerounaises, l'Approvisionnement en Eau Potable (AEP) dans le périmètre urbain de Foumban reste un défi majeur pour les populations. L'objectif de ce travail est de comprendre la dynamique hydrochimique et le niveau de vulnérabilité des eaux de cette zone située dans le bassin versant du Nchi, plateau Bamoun, à l'Ouest du Cameroun. La méthodologie mise en œuvre a consisté d'une part au suivi des paramètres physico-chimiques dans les eaux du cours d'eau Nchi et les eaux de nappes et d'autre part, à la caractérisation des paramètres bactériologiques dans les eaux souterraines sélectionnées en fonction de leur répartition spatiale et de leur sollicitation par les populations. Les résultats montrent que ces eaux sont acides à neutres $(5.3 \leq \mathrm{pH} \leq 7,7)$ et faiblement minéralisées $[(15 \mu \mathrm{S} / \mathrm{cm}<\mathrm{C}$.E $<164,9 \mu \mathrm{S} / \mathrm{cm})$ et $\left.\left(184 \mu \mathrm{eq} / \mathrm{l} \leq \mathrm{TZ}^{+} \leq 1565 \mu \mathrm{eq} / \mathrm{l}\right)\right]$. La concentration moyenne de la silice pondérée par les débits est de 23,55 $\mathrm{mg} / \mathrm{l}$ et représente $59,33 \%$ de la somme de matières dissoutes minérales (TDS) qui est de 39,68 mg/l. L'ordre de grandeur décroissant des éléments majeurs dans les eaux du bassin versant du Nchi est le suivant : $\mathrm{HCO}_{3}^{-}>$ $\mathrm{Ca}^{2+}>\mathrm{Mg}^{2+}>\mathrm{Na}^{+}>\mathrm{K}^{+}>\mathrm{NO}_{3}^{-} \geq \mathrm{Cl}^{-}$traduisant ainsi des eaux bicarbonatées calci-magnésiennes. Les variations spatiales des différentes espèces chimiques montrent de fortes concentrations dans les eaux de nappe par rapport aux eaux d'écoulement. Les analyses statistiques, les variations temporelles des minéraux dissouts et des fortes corrélations inter-élémentaires observées traduisent de leur origine commune qui est surtout l'altération chimique. L'analyse bactériologique met en évidence l'absence des streptocoques fécaux (SF) dans les puits $\mathrm{P} 1$ et $\mathrm{P} 4$ et la présence remarquée des coliformes fécaux (CF) qui sont de $30 \mathrm{UFC} / 100 \mathrm{ml}, 20$ $\mathrm{UFC} / 100 \mathrm{ml}$ et $36 \mathrm{UFC} / 100 \mathrm{ml}$ respectivement dans les puits P1, P4 et la source S1. La présence de ces bactéries thermo-tolérantes, de même que les nitrates fortement représentés dans les eaux souterraines de la ville de Foumban montrent que ces eaux présentent une dégradation liée aux activités humaines.

(C) 2015 International Formulae Group. All rights reserved.

Mots clés: Lithologie, anthropisation, qualité de l'eau, altération chimique, bassin versant du Nchi, plateau Bamoun.

\section{Hydrochemistry and potability of water from the watershed Nchi in Bamoun plateau (West Cameroon)}

\section{ABSTRACT}

As it is the case in most of Cameroonian towns, the Water Supply (WS) in the Foumban urban area remains a major challenge to its inhabitants. The objective of this work is to understand the hydrochemical dynamics and the vulnerability of the water of this area located in the Nchi watershed, Bamoun plateau, West Cameroon. The methodology firstly consisted in the monitoring of physico-chemical parameters in the river 
Nchi and the underground water. Secondly, in the characterization of bacteriological parameters in underground water selected according to their spatial distribution and types frequently used by the population (wells, springs). The results show that the water is acidic to neutral $(5.3 \leq \mathrm{pH} \leq 7.7)$ and weakly mineralized $[(15 \mu \mathrm{S} / \mathrm{cm}<\mathrm{EC}<164.9 \mu \mathrm{S} / \mathrm{cm})$ and $(184 \mu \mathrm{eq} / \mathrm{l} \leq+\leq \mathrm{TZ} 1565 \mu \mathrm{eq} / \mathrm{l})]$. The average concentration of weighted silica by the discharge rate is $23.55 \mathrm{mg} / \mathrm{l}$, representing $59.33 \%$ of the sum of total dissolved solids (TDS) which is $39.68 \mathrm{mg} / \mathrm{l}$. The decreasing order of magnitude of the major elements in the water of the Nchi watershed is: $\mathrm{HCO}_{3}^{-}>\mathrm{Ca}^{2+}>\mathrm{Mg}^{2+}>\mathrm{Na}^{+}>\mathrm{K}^{+}>\mathrm{NO}_{3}^{-} \geq \mathrm{Cl}^{-}$thus reflecting a calco-Mg-bicarbonate water. The spatial variations of different chemical elements show high concentrations in underground water as compared to surface flow. Statistical analysis, temporal variations of dissolved minerals and strong inter-element correlations indicate that they have a common origin, mainly resulting from chemical weathering. Bacteriological analysis shows the absence of faecal streptococci (SF) in the P1 and P4 wells and the significant presence of faecal coliforms (FC) which is $30 \mathrm{CFU} / 100 \mathrm{ml} 20 \mathrm{CFU} / 100 \mathrm{ml}$ and $36 \mathrm{CFU} / 100 \mathrm{ml}$ respectively in the P1, P4 well and the S1 spring. The presence of these heat-tolerant bacteria, as well as nitrates strongly represented in groundwater of the Foumban town, shows that the water is polluted as a result human activities.

Keywords: Lithology, anthropic activities, water quality, chemical weathering, Nchi watershed, Bamoun plateau.

\section{INTRODUCTION}

Les ressources en eau sont rarement pures de manière générale dans la nature ; différents éléments peuvent altérer sa constitution de base par diffusion, dissolution et hydrolyse, ou même par un simple mélange (Savané et Soro, 2001 ; Xiao et al., 2012 ; Yao et al., 2012). La composition de l'eau va dépendre ainsi de l'environnement dans lequel elle se trouve. Les éléments qui se mélangent à cette ressource lui confèrent un chimisme particulier traduisant certaines propriétés ou spécificités intrinsèques à son trajet (Xiao et al., 2012). En traversant donc des couches géologiques plus ou moins perméables, l'eau souterraine est susceptible de présenter une augmentation de sa minéralisation par lessivage des roches, notamment en cas de nappes libres (Andre et al., 2005 ; Adrian et al., 2005). Cette situation peut donner lieu à des atteintes graves à la qualité de l'eau par contamination verticale due aux activités anthropogènes (Yassir et Amira, 2013 ; Omar et al., 2014 ; Ntep et al., 2014).

$\mathrm{Au}$ Cameroun, comme dans la plupart des pays africains en voie de développement, $\mathrm{du}$ fait d'une démographie galopante, d'un manque d'investissement dans le secteur de l'eau notamment pour son traitement et sa distribution, du manque de culture scientifique et d'assainissement, du chevauchement entre les structures chargées de la distribution de cette ressource ajouté aux variabilités et changements climatiques actuels, les populations des grandes métropoles font face aux nombreux problèmes d'approvisionnement en eau de bonne qualité et en quantité suffisante (Yao et al., 2012). Dans la ville de Foumban, la compagnie de distribution des eaux (CDE) dispose des installations qui datent de 1975 ; elle produit seulement $2520 \mathrm{~m}^{3} / \mathrm{j}$, ce qui représentent $1 / 5$ de la demande, sur une population estimée à 83522 habitants (Bucrep, 2005). 4670 points d'eau sont créés avec 3925 abonnés. La population dans sa plus grande majorité a recours aux ouvrages parallèles (puits, sources, lacs, étangs, rivières, pluies, etc.) pour leurs besoins. Cette situation est à l'origine de nombreux cas d'infection des maladies hydriques (fièvre typhoïde, bilharziose, diarrhée). D'après les responsables de l'Hôpital de District de Foumban environ $20 \%$ des cas de diagnostics dans cette formation hospitalière confirment des cas de maladies hydriques allant jusqu'à une certaine endémicité marquée par des résurgences épidémiologiques qui sévissent beaucoup plus en saison sèche. Tous ces pesanteurs palpables concourent et de façon considérable à la paupérisation des populations.

Plusieurs études ont été effectuées au Cameroun, en hydrologie, hydrogéologie, 
hydrogéohimie, et hydrobiologie au cours de la dernière décennie (Njiné et al., 2001 ; Moundi, 2004 ; Moundi et al., 2007 ; Tita, 2008; Mouncherou et al., 2011). Cependant, très peu de travaux ont été effectués dans le bassin versant du Nchi en particulier dans le domaine de la qualité des eaux. Le présent travail a pour objectif l'évaluation des qualités physico-chimiques et bactériologiques des eaux du bassin versant $\mathrm{du}$ Nchi aussi bien les eaux du cours d'eau que celles des nappes contenues dans le bassin.

\section{MATERIEL ET METHODES}

\section{Situation géographique, Contexte géomorphologique, géologique, climatique et hydrologique de la zone d'étude}

Le bassin versant du Nchi est situé dans le département du Noun à l'Ouest-Cameroun (Figure 1). Il couvre une superficie de 1287,92 $\mathrm{km}^{2}$ à son exutoire (confluence avec le Mbam). Son relief qui ressort environ cinq grandes unités géomorphologiques (Figure 2) est constitué de collines et de vallées dont l'ensemble offre un paysage ondulé parfois assimilable à de véritables escarpements. Son substratum montre deux grands ensembles à savoir les roches granito-gneissiques et les basaltes transitionnels (Figures 3) de plateau d'âge précambrien (Moundi, 2004), sur lesquels sont identifiés quatre types de sols dont : les sols ferralitiques épais et majoritaires, les sols hydromorphes, les sols jeunes et les sols minéraux bruts (Njoya, 2007). Le bassin versant du Nchi est caractérisé par un climat tropical de montagne à deux saisons fortement contrastées dont une longue saison pluvieuse ( 9 mois) et une courte saison sèche (3 mois). La végétation est de type savane arbustive, une zone de transition entre le Sud forestier et le Nord-Cameroun. Les précipitations moyennes interannuelles sont de $1855.6 \pm 203 \mathrm{~mm}$. La température moyenne quant à elle est de $21.3^{\circ} \mathrm{C}$.
Sur le plan hydrogéologique, les premières études ont été rendues possibles à partir des travaux préliminaires effectués en vu de l'implantation de quelques forages pour l'alimentation en eau dans la zone. Les rapports issus de ces campagnes permettent de distinguer deux grands aquifères: Les aquifères d'altérites et des fissures. Les premiers, beaucoup plus sollicités par les populations, jouent un rôle capacitif et les seconds un rôle conducteur. Les deux aquifères identifiés fonctionnent en un système bicouche (Maréchal et al., 2003; Lachassagne et Wing, 2005).

\section{Méthodologie}

La méthodologie adoptée dans ce travail a consisté au suivi des paramètres physico-chimiques des eaux superficielles du cours d'eau Nchi, et des eaux souterraines de nappe et à la caractérisation des paramètres bactériologiques dans les eaux de nappe. Les travaux sont effectués aussi bien sur le terrain qu'en laboratoire.

Sur le terrain, il a été procédé au choix et à la description des points de prélèvement (Tableau 1), à la mesure in situ des paramètres physico-chimiques notamment, le $\mathrm{pH}$ à l'aide d'un appareil de marque «WTW pH 315 i/ SET» couplé à une électrode de marque Sen Tix41 WTW qui est conservée dans une solution de chlorure de potassium $(\mathrm{KCl})$ dont la concentration est de $3 \mathrm{~mol} / \mathrm{l}$.

La conductivité et la température quant à elles ont été mesurées simultanément à l'aide d'un conductimètre de marque «WTW cond330i/ SET» couplé également à une électrode Tetra con ${ }^{\mathrm{R}}$ WTW 325. L'appareil est préalablement calibré par une solution étalon d'une conductivité de $1413 \mu \mathrm{S} / \mathrm{cm}$ à $25^{\circ} \mathrm{C}$. La précision est de $0,1 \mu \mathrm{s} / \mathrm{cm}$ pour la conductivité et $0,1{ }^{\circ} \mathrm{C}$ pour la température. Les échantillons d'eau du cours d'eau Nchi, de puits et de source ont été prélevés dans des bouteilles en polyéthylène, préalablement lavées à l'eau 
distillée et à celle de l'échantillonnage, puis conditionnés pour les analyses en laboratoire.

Les travaux de laboratoire ont consisté à l'analyse des paramètres chimiques (éléments majeurs, aluminium et fer total, silice dissoute) dans le cours d'eau Nchi et les eaux souterraines selon les méthodes appropriées (Rodier, 2009), à la caractérisation des paramètres bactériologiques (coliformes et streptocoques fécaux) par la technique de filtration sur membrane (Rodier, 2009). Cette dernière a été réalisée dans les eaux souterraines (source et puits) sélectionnées en fonction de leur répartition spatiale et de leur sollicitation par les populations riveraines. La période de mesures s'est étalée sur huit mois, entre le 23 janvier 2009 au 19 février 2010 et couvrant une année hydrologique. L'analyse hydrochimique a été réalisée par le logiciel Diagramme. Le traitement statistique multivarié a aussi été réalisé. C'est la méthode d'analyse en composantes principales qui a été appliquée. En effet, cette méthode est souvent utilisée dans plusieurs domaines, notamment en sciences de l'eau (Yao et al., 2012 ; Eblin et al., 2014). Son objectif est la description des liaisons entre deux variables par des techniques statistiques bidimensionnelles conduisant à se poser la question de la représentation simultanée de données en dimension plus grande que 2 (Moore et al., 2009).

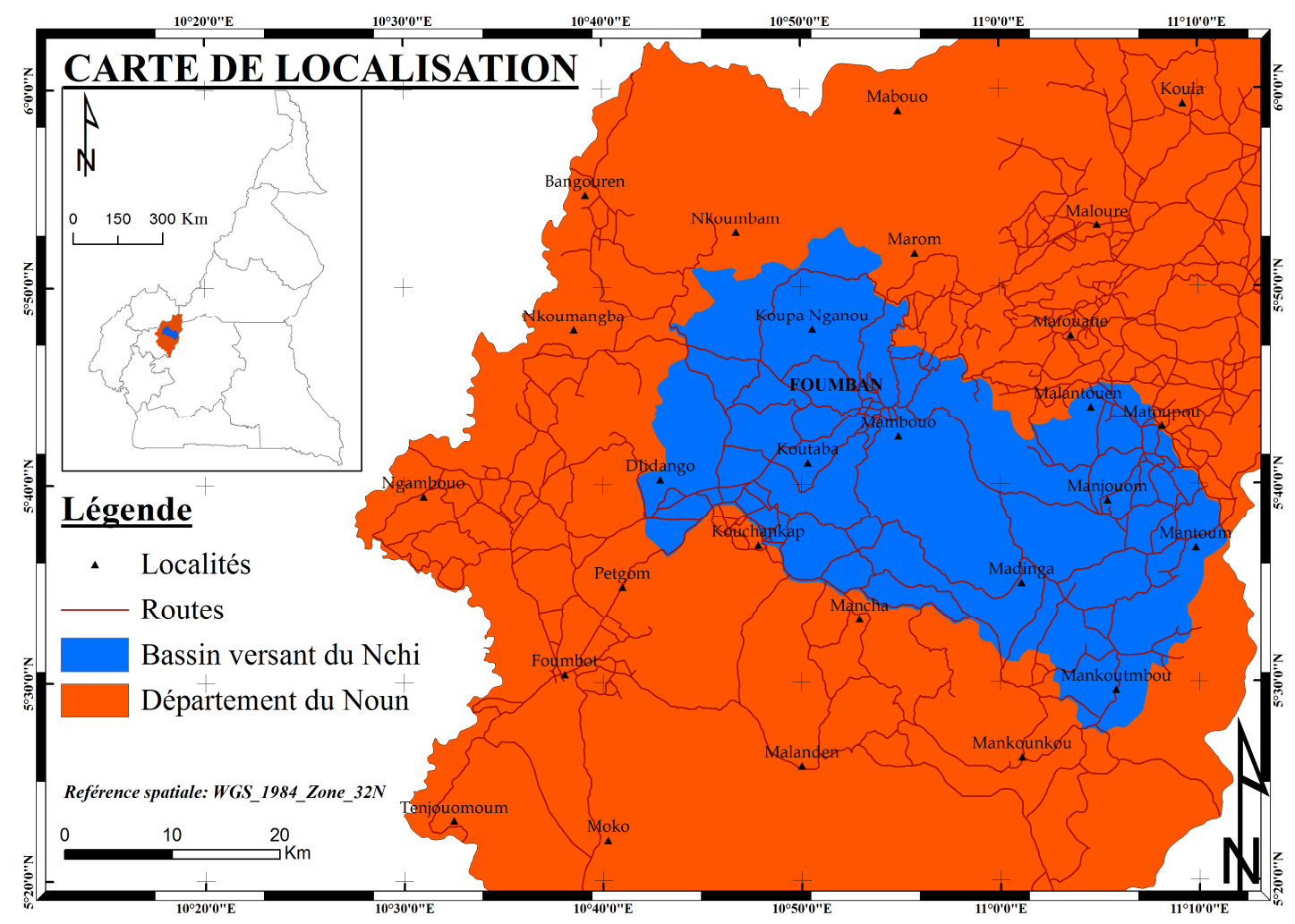

Figure. 1 : Situation géographique du bassin versant du Nchi. 


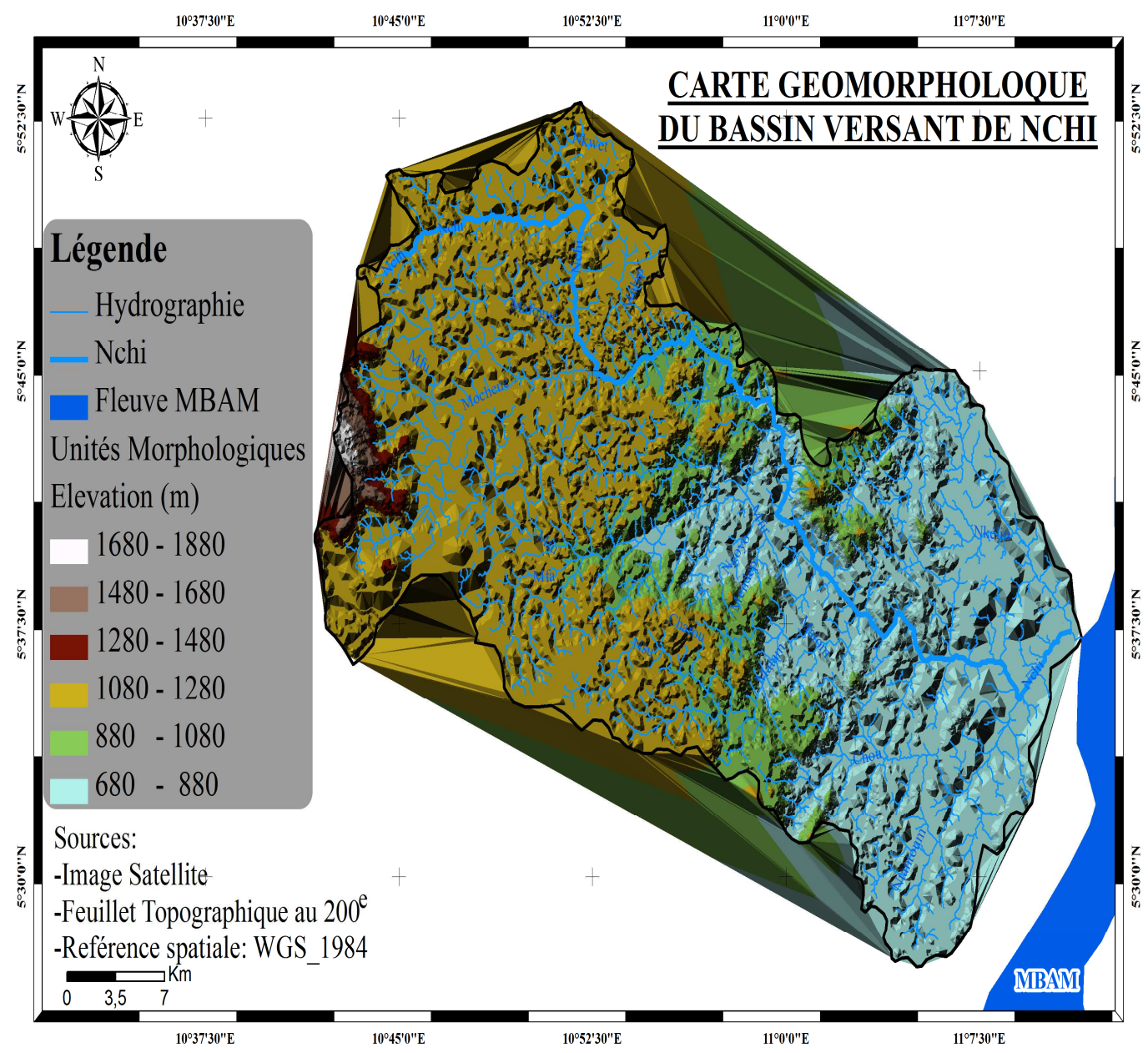

Figure 2 : Les grandes unités géomorphologiques du bassin versant du Nchi en 3D.
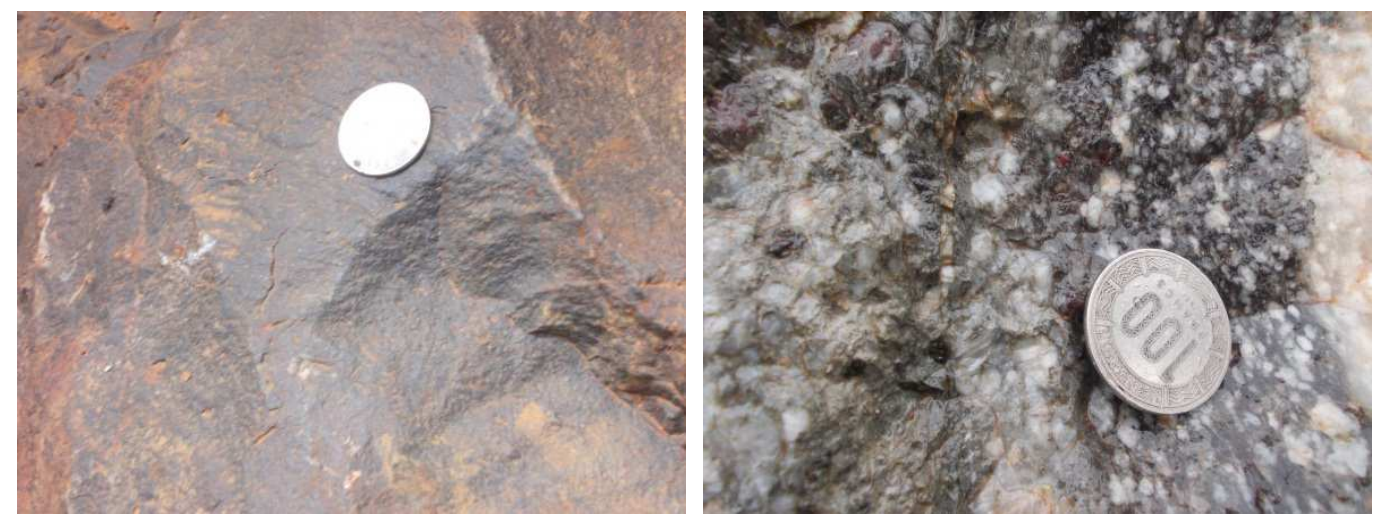

Figures 3 : Quelques formations géologique qui affleurent dans la zone d'étude (secteur Njiloum et Njindaré à Foumban). 
Tableau 1 : Coordonnées géographiques des points d'adduction d'eau analysés à Foumban (puits, sources, rivière).

\begin{tabular}{|c|c|c|c|}
\hline Points identifiés & $\mathbf{X}$ & $\mathbf{Y}$ & $\mathbf{Z}(\mathbf{m})$ \\
\hline $\mathbf{P}_{1}$ & N05 ${ }^{\circ} 44^{\prime} 23^{\prime \prime}$, & E10 $53^{\circ} 03^{\prime \prime}$ & 1143 \\
\hline $\mathbf{P}_{4}$ & N05 $44^{\prime} 22^{\prime \prime}$ & $\mathrm{E} 10^{\circ} 53^{\prime} 20^{\prime \prime}$ & 1134 \\
\hline $\mathbf{S}_{1}$ & N05 ${ }^{\circ} 44^{\prime} 00^{\prime \prime}$ & E10 53’03” & 1175 \\
\hline Nchi & $\mathrm{N}^{\circ} 5^{\circ} 44^{\prime} 45^{\prime \prime}$ & $\mathrm{E} 10^{\circ} 52^{\prime} 58^{\prime \prime}$ & 1094 \\
\hline
\end{tabular}

\section{RESULTATS}

Niveau de variation des paramètres physico-chimiques et chimiques

Le $\mathrm{pH}$ des eaux du bassin versant du Nchi se situe entre 5,3 et 7,7 alors que les valeurs de la conductivité électrique (C.E) sont comprises entre $15,3 \mu \mathrm{S} / \mathrm{cm}$ et 164,9 $\mu \mathrm{S} / \mathrm{cm}$ (Tableaux 2 et 3 ). Ces valeurs varient très peu entre les eaux souterraines (puits et source) et les eaux d'écoulement du Nchi (Figure 4). Par contre, la conductivité électrique (C.E) présente des variations significatives; elle est plus élevée dans les eaux de nappe contrairement aux eaux de surface. Les résultats statistiques des paramètres chimiques obtenus au cours de la période d'étude dans le cours d'eau Nchi et les résultats de l'analyse ponctuelle des eaux de nappes (source et puits) sont consignés dans les Tableaux 2 et 3 respectivement. Les variations spatiales des éléments chimiques dans les eaux du bassin versant du Nchi (Figure 4) montrent que les alcalino-terreux $\left(\mathrm{Ca}^{2+}, \mathrm{Mg}^{2+}\right)$ présentent des concentrations plus élevées dans la source $S_{1}$ et le puits $P_{1}$; et sont pratiquement dans la même gamme de valeurs dans le puits $\mathrm{P}_{4}$ et le cours d'eau Nchi.

Les alcalins $\left(\mathrm{Na}^{+}\right.$et $\left.\mathrm{K}^{+}\right)$par contre n'ont pas la même évolution; le potassium a une forte concentration dans la source, suivi du cours d'eau Nchi. Les puits $\mathrm{P}_{1}$ et $\mathrm{P}_{4}$ ont pratiquement la même concentration. Quant au sodium, les fortes teneurs sont observées dans la source $S_{1}$; les puits $P_{1}$ et $P_{2}$ ont des valeurs sensiblement égales mais supérieures à celle du cours d'eau Nchi.

La somme des équivalents cations $\left(\mathrm{TZ}^{+}\right)$est alors beaucoup plus représentée dans la source suivi du puits $\mathrm{P}_{1}$, elle est du même ordre de grandeur dans le puits $\mathrm{P}_{4}$ et le cours d'eau Nchi. Les bicarbonates sont fortement représentés dans la source $S_{1}$ suivi du puits $P_{1}$. Le puits $\mathrm{P}_{4}$ et le cours d'eau Nchi ont des teneurs sensiblement égales (Figure 4).

Les concentrations en nitrates sont du même ordre de valeur dans le puits $P_{1}$ et la source $S_{1}$; cette concentration est par contre forte dans le puits $\mathrm{P}_{4}$. Dans le cours d'eau Nchi, les nitrates sont très peu représentés et se retrouvent sous forme de traces.

Les concentrations en chlorures sont du même ordre de grandeur dans les puits $\mathrm{P}_{4}$ et le cours d'eau Nchi ; cette concentration est très élevée dans la source $S_{1}$, mais faible dans le puits $\mathrm{P}_{1}$. La TZ (somme des équivalents anions) a une forte concentration dans la source suivie des puits $\mathrm{P}_{1}$, et $\mathrm{P}_{4}$ et du cours d'eau Nchi (Figure 4). Ces différentes fluctuations montrent que les éléments dissouts pour la plupart sont en général beaucoup plus concentrés dans les eaux souterraines (sources, puits) par rapport aux eaux d'écoulement (cours d'eau Nchi).

\section{Typologie des eaux du bassin versant du Nchi}

Durant la période d'étude, l'ordre de grandeur décroissant des cations majeurs par rapport à la $\mathrm{TZ}^{+}$dans le cours d'eau Nchi est le suivant: $\mathrm{Ca}^{2+}(33,14 \%)>\mathrm{Na}^{+}(24,21 \%)>$ $\mathrm{Mg}^{2+}(22,42 \%)>\mathrm{K}^{+}(20,22 \%)$, et celui des anions par rapport à la $\mathrm{TZ}^{-}$est: $\mathrm{HCO}_{3}^{-}(85,51$ $\%)>\mathrm{Cl}^{-}(10,11 \%)>\mathrm{NO}_{3}^{-}(1,76 \%)$.

Pour ce qui est des eaux de la source $\mathrm{S}_{1}$, l'ordre de grandeur décroissant des cations est de: $\mathrm{Ca}^{2+}(44,08 \%)>\mathrm{Mg}^{2+}(35,40 \%)>$ $\mathrm{Na}^{+}(18,22 \%)>\mathrm{K}^{+}(2,29 \%)$, cet ordre pour les anions est: $\mathrm{HCO}_{3}^{-}(77,09 \%)>\mathrm{Cl}^{-}(11,87$ $\%)>\mathrm{NO}_{3}{ }^{-}(11,03 \%)$. Les eaux du puits $\mathrm{P}_{1}$, ont un ordre décroissant des cations de: $\mathrm{Ca}^{2+}$ 
$(43,12 \%)>\mathrm{Mg}^{2+}(31,88 \%)>\mathrm{Na}^{+}(22,55 \%)$ $>\mathrm{K}^{+}(6,60 \%)$; alors que pour les anions, on a: $\mathrm{HCO}_{3}^{-}(80,56 \%)>\mathrm{NO}_{3}^{-}(17,18 \%)>\mathrm{Cl}^{-}$ $(2,25 \%)$. Dans le puits $\mathrm{P}_{4}$, l'ordre décroissant des éléments est de: $\mathrm{Na}^{+}(53,08 \%)>\mathrm{Ca}^{2+}$ $(24,14 \%) \mathrm{Mg}^{2+}(15,67 \%)>\mathrm{K}^{+}(7,17 \%)$; et enfin les proportions des anions sont les suivantes: $\mathrm{NO}_{3}{ }^{-}(54,49 \%)>\mathrm{HCO}_{3}{ }^{-}(39,90 \%)$ $>\mathrm{Cl}^{-}(5,62 \%)$.

L'ordre général décroissant des principaux éléments chimiques dans le cours d'eau Nchi par rapport à la $\mathrm{TZ}^{+}$et la $\mathrm{TZ}^{-}$est le suivant: $\mathrm{HCO}_{3}^{-}>\mathrm{Ca}^{2+}>\mathrm{Na}^{+}>\mathrm{Mg}^{2+}>\mathrm{K}^{+}>$ $\mathrm{Cl}^{-}>\mathrm{NO}_{3}{ }^{-}$; cet ordre pour les eaux de source $\mathrm{S}_{1}$ est: $\mathrm{HCO}_{3}^{-}>\mathrm{Ca}^{2+}>\mathrm{Mg}^{2+}>\mathrm{Na}^{+}>\mathrm{NO}_{3}{ }^{-}>$ $\mathrm{Cl}^{-}>\mathrm{K}^{+}$; il est de: $\mathrm{Ca}^{2+}>\mathrm{HCO}_{3}^{-}>\mathrm{Mg}^{2+}>$ $\mathrm{Na}^{+}>\mathrm{NO}_{3}^{-}>\mathrm{Cl}^{-} \geq \mathrm{K}^{+}$pour les eaux du puits $\mathrm{P}_{1}$ et enfin $\mathrm{NO}_{3}^{-}>\mathrm{HCO}_{3}^{-}>\mathrm{Na}^{+}>\mathrm{Ca}^{2+}>$ $\mathrm{Mg}^{2+}>\mathrm{Cl}^{-}>\mathrm{K}^{+}$pour les eaux du puits $\mathrm{P}_{4}$.

Les eaux du bassin versant du Nchi pour la plupart sont alors de faciès bicarbonaté calcique-magnésien (Figure 5). Le puits $\mathrm{P}_{4}$, situé en zone de marécage et en contact permanent avec la rivière Ngâh qui traverse les quartiers Njintout et Njiloum, se démarque de cette logique et présente plutôt un faciès non bicarbonaté.

Variation saisonnière des paramètres physico-chimiques dans le cours d'eau Nchi Les espèces minérales dissoutes (cations majeurs, anions majeurs, silices) présentent, pour la plupart, des concentrations élevées en saison sèche (janvier et février) et faibles pendant la saison pluvieuse (mars, avril, mai, juin et août) (Figure 6).

Certains de ces éléments chimiques à l'instar du calcium, magnésium, potassium, bicarbonates, ont plutôt des concentrations élevées en début de saison pluvieuse (mars).

\section{Variation spatiale des paramètres bactériologiques dans les eaux de nappe du bassin versant du Nchi}

La Figure 9 montre que les eaux des puits $\mathrm{P}_{1}$ et $\mathrm{P}_{4}$ sont exempts des streptocoques fécaux. La source $S_{1}$ par contre est contaminée tant par les coliformes fécaux (CF) que les streptocoques fécaux $(\mathrm{SF})$.

Tableau 5: Normes en paramètres physico-chimiques et bactériologiques pour les eaux de consommation (Gilli et al., 2008).

\begin{tabular}{lccc}
\hline Eléments & Teneurs & Limite OMS & Unité \\
\hline $\mathrm{CF}$ & $20-36$ & 0 & $\mathrm{UFC} / 100 \mathrm{ml}$ \\
$\mathrm{SF}$ & $0-33$ & 0 & $\mathrm{UFC} / 100 \mathrm{ml}$ \\
$\mathrm{Ca}^{2+}$ & $0,56-13,8$ & 500 & $\mathrm{mg} / \mathrm{l}$ \\
$\mathrm{Mg}^{2+}$ & $0,26-6,6$ & 50 & $\mathrm{mg} / \mathrm{l}$ \\
$\mathrm{Na}^{+}$ & $0,4-6,5$ & 200 & $\mathrm{mg} / \mathrm{l}$ \\
$\mathrm{K}^{+}$ & $0,32-1,4$ & 12 & $\mathrm{mg} / \mathrm{l}$ \\
$\mathrm{HCO}_{3}{ }^{-}$ & $4,98-49,2$ & - & $\mathrm{mg} / 1$ \\
$\mathrm{Cl}^{-}$ & $0,35-4,3$ & 250 & $\mathrm{mg} / 1$ \\
$\mathrm{SO}_{4}{ }^{2-}$ & 0,00 & 250 & $\mathrm{mg} / 1$ \\
$\mathrm{NO}_{3}{ }^{-}$ & $1,70-14,9$ & 50 & $\mathrm{mg} / 1$ \\
$\mathrm{pH}$ & $5,25-7,90$ & $6,5-9$ & - \\
$\mathrm{T}^{\circ} \mathrm{C}$ & $23-26$ & 25 & ${ }^{\circ} \mathrm{C}$ \\
$\mathrm{C} . \mathrm{E}$. & $15-160$ & $180-1000$ & $\mu \mathrm{S} / \mathrm{cm} \mathrm{à20}{ }^{\circ} \mathrm{C}$ \\
$\mathrm{Fe}$ total & 1,02 & 200 & $\mu \mathrm{gg} / \mathrm{l}$ \\
$\mathrm{Al}$ total & 15,40 & 200 & $\mu \mathrm{gg} / \mathrm{l}$ \\
$\mathrm{TDS}$ & $18,66-46,70$ & 1000 & $\mathrm{mg} / \mathrm{l}$ \\
$\mathrm{Ni}$ & & 0,002 & $\mathrm{mg} / \mathrm{l}$ \\
$\mathrm{Mn}$ & & 0,5 & $\mathrm{mg} / \mathrm{l}$ \\
$\mathrm{Cu}$ & & 2 & $\mathrm{mg} / \mathrm{l}$ \\
$\mathrm{Zn}$ & & 3 & $\mathrm{mg} / 1$ \\
\hline
\end{tabular}


Tableau 2 : Domaine de variations des paramètres physico chimiques dans le cours d'eau Nchi durant la période d'étude (2009).

\begin{tabular}{|c|c|c|c|c|c|c|c|c|c|c|c|c|c|}
\hline & $\begin{array}{l}\mathrm{Ca}^{2+} \\
\mu \mathrm{eq} / \mathrm{l}\end{array}$ & $\begin{array}{l}\mathrm{Mg}^{2+} \\
\mu \mathrm{eq} / \mathrm{l}\end{array}$ & $\begin{array}{c}K^{+} \\
\mu e q / 1\end{array}$ & $\begin{array}{c}\mathrm{Na}^{+} \\
\mu \mathrm{eq} / \mathrm{l}\end{array}$ & $\begin{array}{c}\mathrm{Fe} \\
\mathrm{mg} / \mathrm{l}\end{array}$ & $\begin{array}{c}\text { Al } \\
\mathrm{mg} / \mathrm{l}\end{array}$ & $\begin{array}{c}\mathrm{HCO}_{3}^{-} \\
\mu e q / \mathbf{l}\end{array}$ & $\begin{array}{c}\mathrm{Cl}^{-} \\
\mu \mathrm{eq} / \mathrm{l}\end{array}$ & $\begin{array}{l}\text { NO3- } \\
\mu \mathrm{eq} / \mathrm{l}\end{array}$ & $\begin{array}{l}\mathrm{SiO}_{2} \\
\mathrm{mg} / \mathrm{l}\end{array}$ & $\begin{array}{c}\mathrm{TZ}^{+} \\
\mu \mathrm{eq} / \mathrm{l}\end{array}$ & $\begin{array}{c}\mathrm{TZ} \\
\mu \mathrm{eq} / \mathrm{l}\end{array}$ & $\begin{array}{l}\text { TDS } \\
\text { mg/l }\end{array}$ \\
\hline Min & 28,00 & 21,40 & 8,18 & 17,39 & 0,22 & 9,00 & 81,64 & 9,85 & 1,70 & 10,00 & 87,91 & 104,10 & 18,66 \\
\hline Max & 80,00 & 62,55 & 61,38 & 95,65 & 2,33 & 29,00 & 343,77 & 21,66 & 4,52 & 25,00 & 299,58 & 365,43 & 46,70 \\
\hline Med & 40,00 & 29,63 & 20,20 & 28,70 & 0,93 & 13,00 & 124,59 & 20,00 & 3,11 & 15,50 & 134,10 & 154,03 & 28,72 \\
\hline E.T. & 18,97 & 13,01 & 19,23 & 25,12 & 0,65 & 6,55 & 82,28 & 5,40 & 1,98 & 7,29 & 66,35 & 86,45 & 10,59 \\
\hline Var. & 360,03 & 169,35 & 369,60 & 630,82 & 0,42 & 43,00 & 6769,76 & 29,20 & 3,93 & 53,07 & 4402,30 & 7473,32 & 112,11 \\
\hline $\mathrm{N}$ & 8 & 8 & 8 & 8 & 8 & 8 & 8 & 4 & 2 & 8 & 8 & 8 & 8 \\
\hline
\end{tabular}

Tableau 3 : Concentrations ponctuelles des éléments chimiques dans les eaux des puits et de résurgence de la zone d'étude.

\begin{tabular}{cccccccccccc}
\hline & $\mathbf{p H}$ & $\begin{array}{c}\mathbf{C . E} \\
\boldsymbol{\mu S} / \mathbf{c m}\end{array}$ & $\begin{array}{c}\mathbf{C a}^{\mathbf{2 +}} \\
\boldsymbol{\mu e q} / \mathbf{l}\end{array}$ & $\begin{array}{c}\mathbf{M g}^{2+} \\
\boldsymbol{\mu e q} / \mathbf{l}\end{array}$ & $\begin{array}{c}\mathbf{K}^{+} \\
\boldsymbol{\mu e q} / \mathbf{l}\end{array}$ & $\begin{array}{c}\mathbf{N a}^{+} \\
\boldsymbol{\mu e q} / \mathbf{l}\end{array}$ & $\begin{array}{c}\mathbf{H C O}_{\mathbf{3}}^{-} \\
\boldsymbol{\mu e q} / \mathbf{l}\end{array}$ & $\begin{array}{c}\mathbf{C l}^{-} \\
\boldsymbol{\mu e q} / \mathbf{l}\end{array}$ & $\begin{array}{c}\mathbf{N O}_{\mathbf{3}}^{-} \\
\boldsymbol{\mu e q} / \mathbf{l}\end{array}$ & $\begin{array}{c}\mathbf{T Z}^{+} \\
\boldsymbol{\mu e q} / \mathbf{l}\end{array}$ & $\begin{array}{c}\mathbf{T Z}^{-} \\
\boldsymbol{\mu e q} / \mathbf{l}\end{array}$ \\
\hline $\mathbf{S}_{\mathbf{1}}$ & 6,17 & 164,90 & 690,00 & 554,17 & 35,90 & 285,22 & 806,89 & 124,29 & 115,48 & 1565,00 & 1046,65 \\
$\mathbf{P}_{\mathbf{1}}$ & 5,91 & 54,80 & 234,50 & 173,33 & 13,30 & 122,65 & 490,00 & 13,71 & 104,52 & 543,78 & 608,23 \\
$\mathbf{P}_{\mathbf{4}}$ & 5,25 & 20,10 & 47,50 & 30,83 & 14,10 & 104,35 & 176,56 & 24,86 & 241,13 & 196,78 & 442,54 \\
\hline \multicolumn{10}{c}{}
\end{tabular}


Tableau 4 : Matrice de corrélation entre les paramètres physico-chimiques des eaux de surface du bassin versant du Nchi.

Matrice de corrélation (Pearson) :

\begin{tabular}{|c|c|c|c|c|c|c|c|c|c|c|c|}
\hline Variables & $\mathrm{pH}$ & cond & $\mathrm{Ca} 2+$ & $\mathrm{Mg} 2+$ & $\mathrm{K}+$ & $\mathrm{Na}+$ & $\mathrm{HCO}-$ & NO3- & $\mathrm{Cl}-$ & $\mathrm{TZ}+$ & TZ- \\
\hline $\mathrm{pH}$ & 1 & & & & & & & & & & \\
\hline cond & 0,329 & 1 & & & & & & & & & \\
\hline $\mathrm{Ca} 2+$ & 0,381 & 0,998 & 1 & & & & & & & & \\
\hline Mg2+ & 0,385 & 0,998 & 1,000 & 1 & & & & & & & \\
\hline $\mathrm{K}+$ & 0,910 & 0,417 & 0,446 & 0,458 & 1 & & & & & & \\
\hline $\mathrm{Na}+$ & 0,189 & 0,986 & 0,973 & 0,974 & 0,327 & 1 & & & & & \\
\hline $\mathrm{HCO}-$ & 0,342 & 0,968 & 0,977 & 0,973 & 0,326 & 0,930 & 1 & & & & \\
\hline NO3- & $-0,893$ & 0,023 & $-0,042$ & $-0,041$ & $-0,654$ & 0,184 & $-0,076$ & 1 & & & \\
\hline $\mathrm{Cl}-$ & 0,379 & 0,945 & 0,933 & 0,940 & 0,580 & 0,948 & 0,836 & 0,048 & 1 & & \\
\hline $\mathrm{TZ}+$ & 0,349 & 1,000 & 0,999 & 0,999 & 0,431 & 0,982 & 0,971 & 0,000 & 0,942 & 1 & \\
\hline TZ- & 0,084 & 0,965 & 0,953 & 0,950 & 0,167 & 0,981 & 0,949 & 0,234 & 0,867 & 0,961 & 1 \\
\hline
\end{tabular}

Cations/anions majeurs, $(\mu \mathrm{eq} / 1) ; \mathbf{T Z}^{+}$et $\mathbf{T Z}(\mu \mathrm{eq} / \mathrm{l})$,
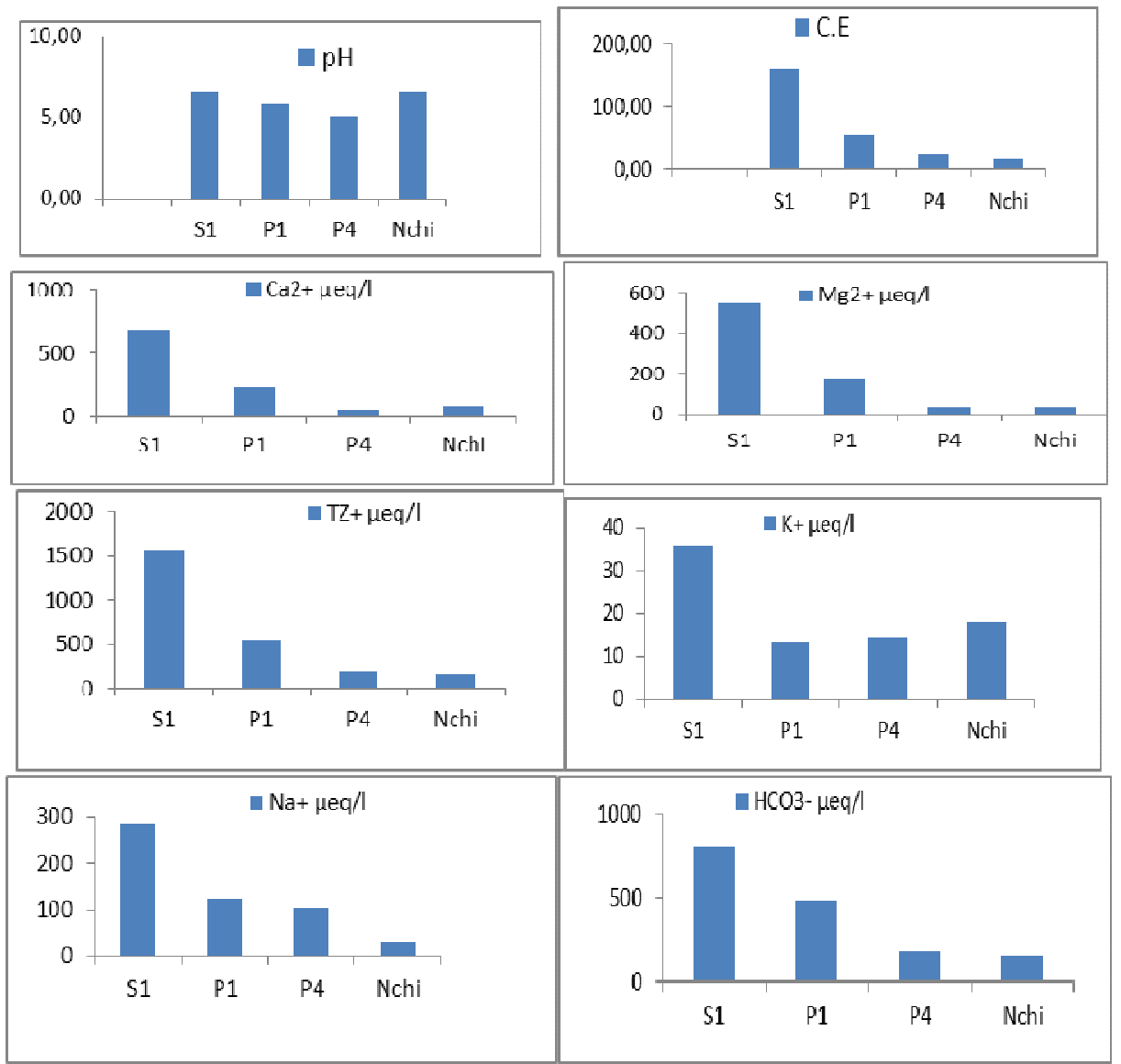


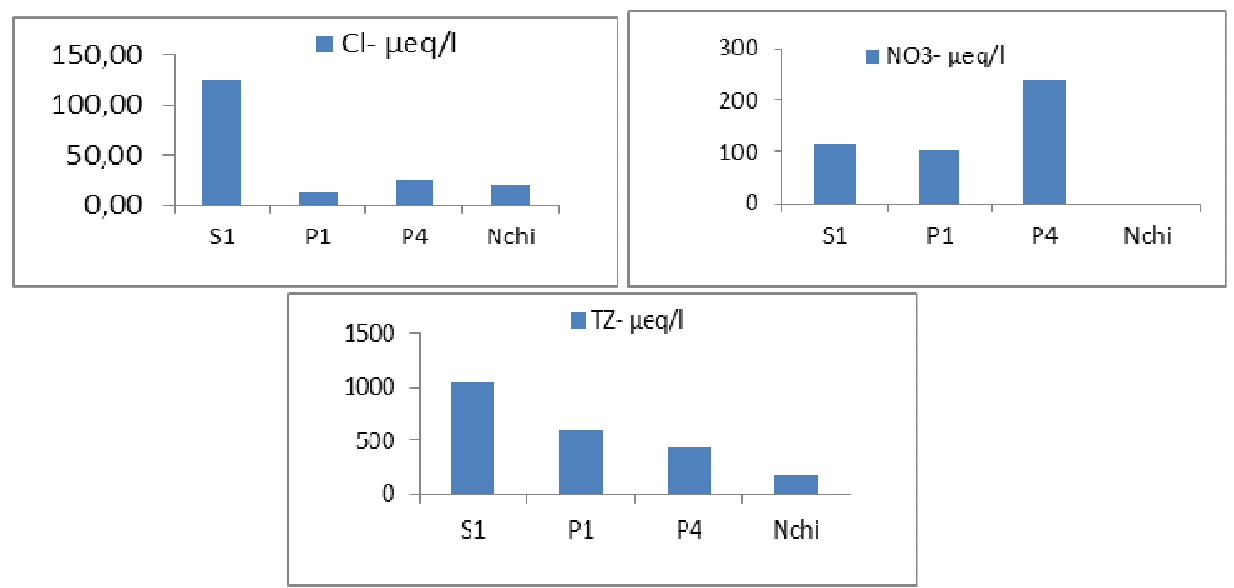

Figure 4: Variation spatiale des éléments chimiques dans les eaux du bassin versant du Nchi.

\section{Faciès hydrochimique des eaux du bassin versant du Nchi}

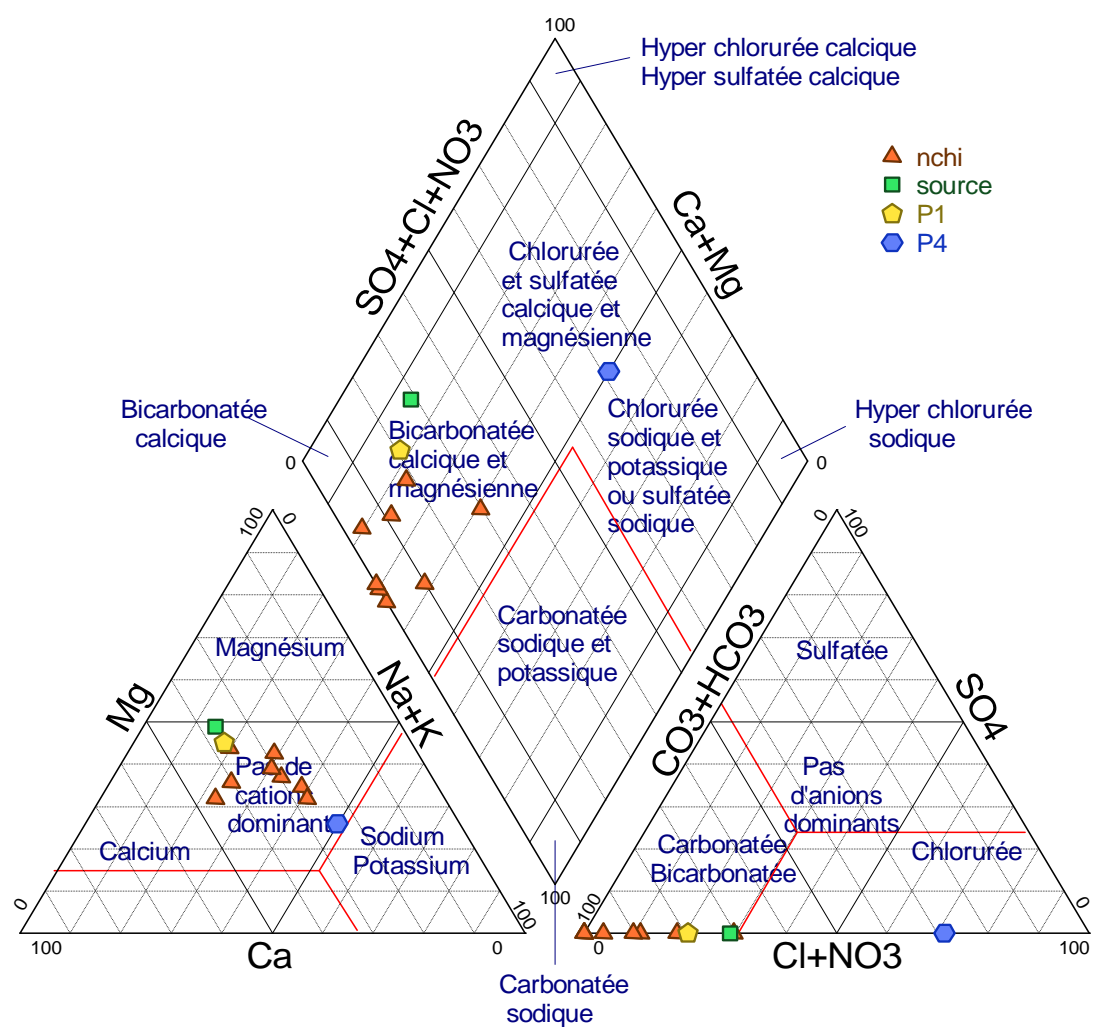

Figure 5 : Diagramme de Piper des eaux du bassin versant du Nchi. 

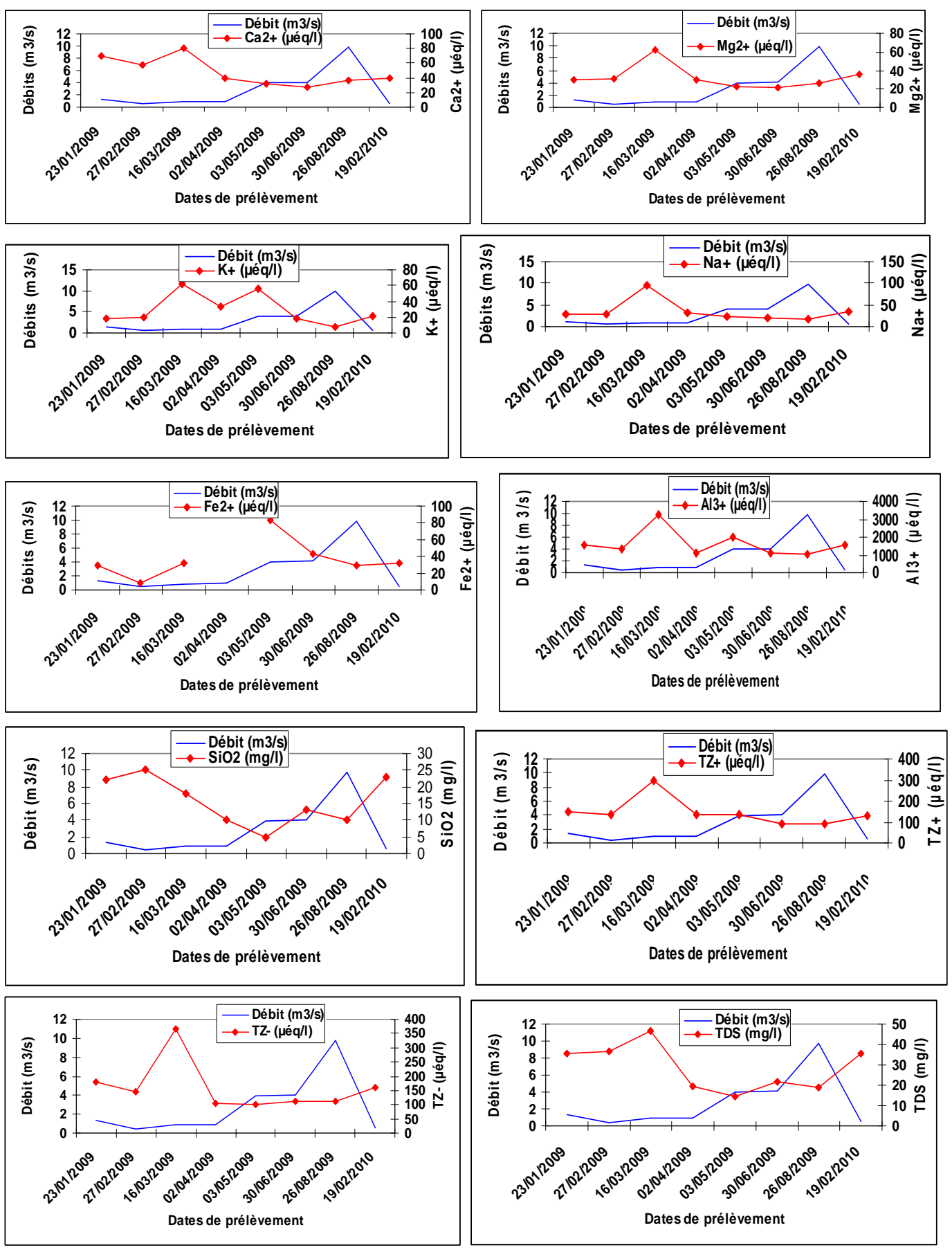

Figure 6: Variations saisonnières des éléments chimiques dissouts dans les eaux du Nchi. 


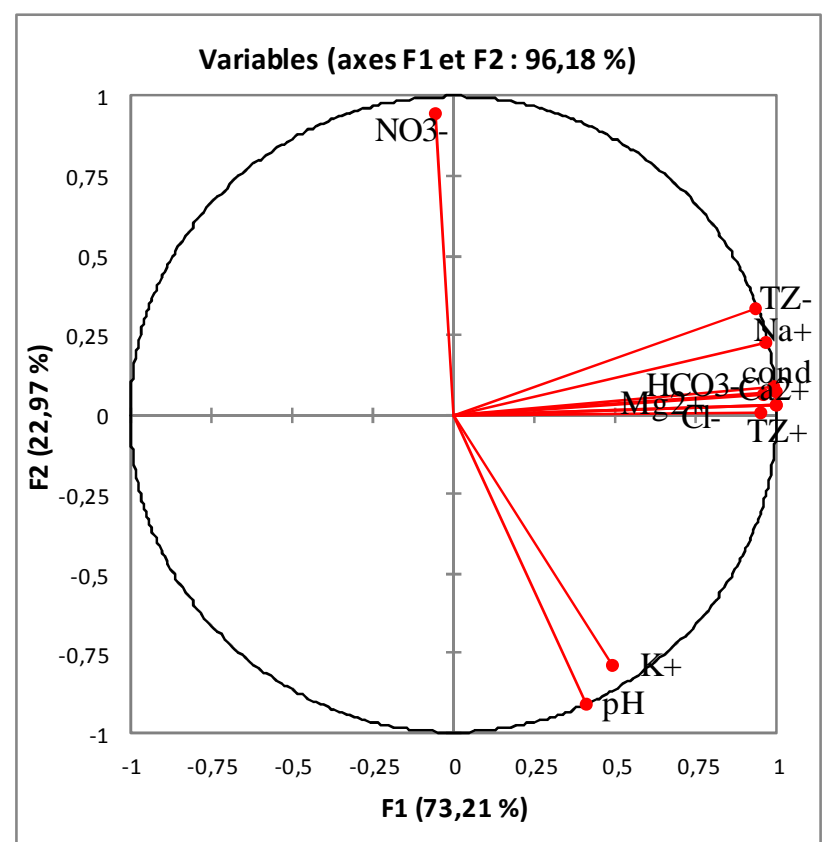

Figure 7: Analyse dans le plan factoriel F1-F2.

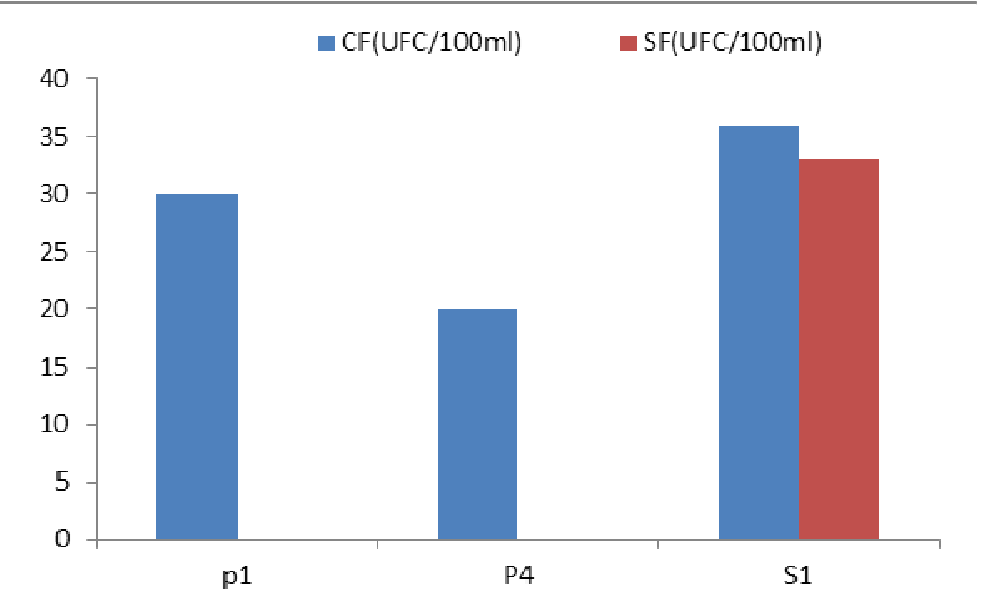

Figure 9: Variation des paramètres bactériologiques dans les eaux souterraines du bassin versant du Nchi. 

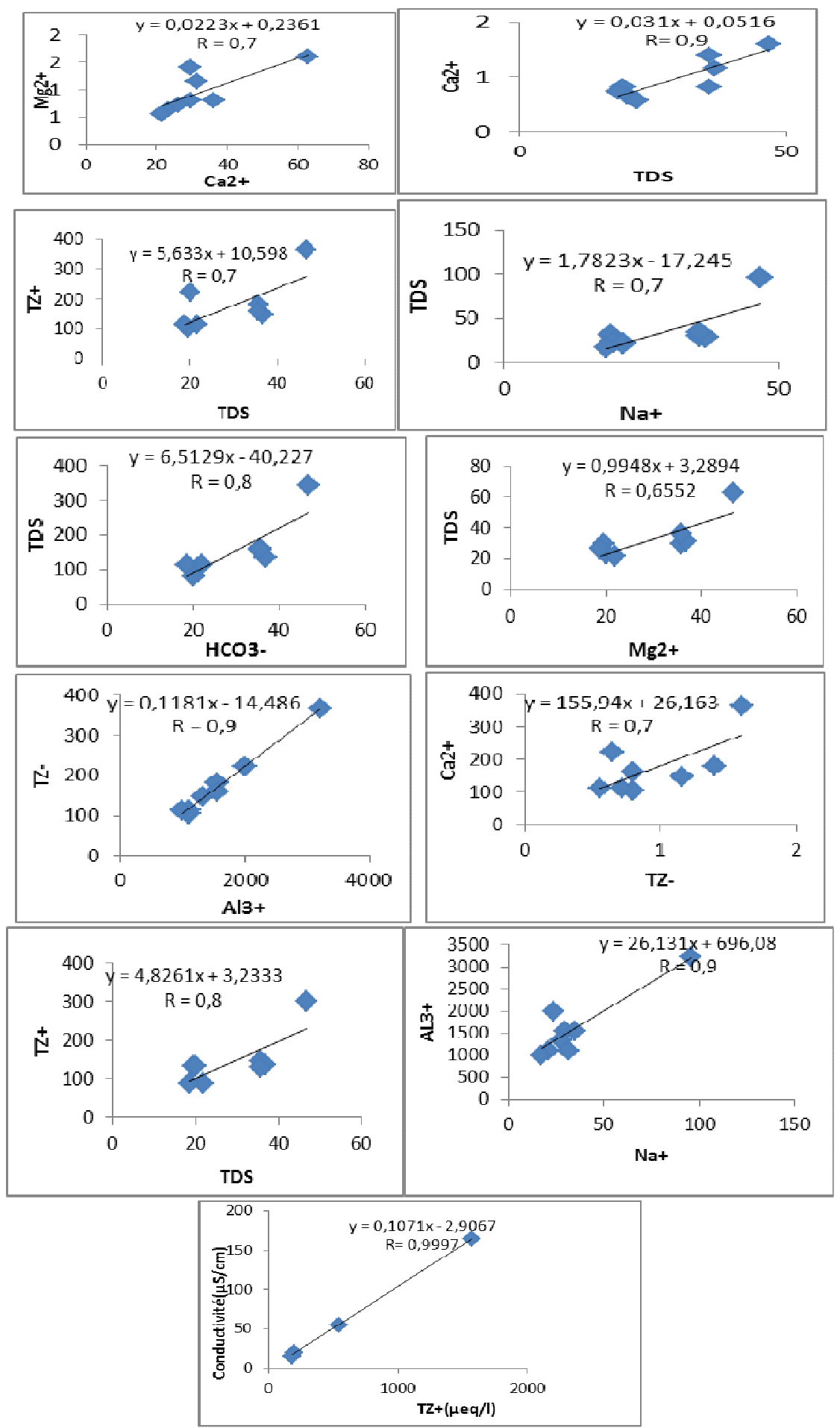

Figure 8: Droites de corrélation entre les différentes espèces chimiques dissoutes dans les eaux du cours d'eau Nchi. 


\section{DISCUSSION}

\section{Spécificité des eaux du bassin versant du} Nchi

Les valeurs des paramètres physiques ( $\mathrm{pH}$ et $\mathrm{CE}$ ) des eaux du bassin versant du Nchi montrent que ces dernières sont acides à neutres, faiblement minéralisées (Rodier, 2009). Elles sont du même ordre de grandeur que celles trouvées par Xiao et al. (2012) dans le même substratum en Chine, Ntep et al. (2014) dans le bassin versant de la Biyémé à Yaoundé, Kouassi et al. (2012) dans le bassin versant du N'zi en Côte d'Ivoire. Les conductivités enregistrées dans le cours d'eau Nchi sont inférieures à celles obtenues par Tita (2008) $(56,1 \leq$ C.E $\leq 349,9 \mu \mathrm{S} / \mathrm{cm})$ dans le Nkoup à Foumbot situé en zone volcanique d'âge récent (quaternaire). Ces remarquent montrent que la signature chimique des eaux dépend étroitement de la formation géologique qu'elle draine (Andre et al., 2005 ; Xing et al., 2013).

Cette minéralisation des eaux est le résultat de deux phénomènes : la dissolution par hydrolyse acide des minéraux de la roche et le mécanisme d'oxydoréduction (Savané et Soro, 2001; Eblin et al., 2014). Cette minéralisation observée dans les eaux de nappe est également liée au temps de résidence beaucoup plus long de ces eaux avec le substratum rocheux et les sols.

Quant aux variations saisonnières, les multiples fluctuations constatées sont dues à des phénomènes de dilution pendant la période pluvieuse et d'évaporation durant la période d'étiage (Lallahem, 2002). En effet, pendant la période d'étiage, les eaux d'écoulement sont alimentées en grande partie par les nappes. Ces dernières fonctionnent comme des réservoirs beaucoup plus chargés, ceci grâce aux propriétés d'éponge des pores des roches. Par contre, pendant la période de crue, les eaux d'écoulement sont alimentées par les pluies et qui sont moins concentrées en substances dissoutes. Il est à noter une complexité avec le fer, l'aluminium et les chlorures pour qui les concentrations sont fortes en saison pluvieuse.

Etude statistique multi-variée et origines des substances dissoutes dans les eaux du bassin versant du Nchi

Dans le but de comprendre le fonctionnement hydro-chimique du système hydrologique étudié, les données physicochimiques ont fait l'objet d'une étude statistique multivariée à partir de l'analyse en composantes principales (ACP). L'analyse des variables de l'ACP dans le plan factoriel F1F2 est présentée par la Figure 7. Elle montre que ce plan factoriel représente $96,18 \%$ de la variance exprimée. Le facteur F1 est le plus important, avec $73,21 \%$ de la variance exprimée, soit plus de la moitié de la variance. Ce graphe met en évidence trois grands regroupements des paramètres étudiés dans les points d'eau. Ces paramètres sont soient confondus, soient tout proches du cercle et donc sont très bien représentés. Le premier regroupement qui prend en compte $\mathrm{pH}$ et $\mathrm{K}^{+}$ traduit le phénomène de pluvio-lessivage des sols. Le deuxième regroupement qui contient $\mathrm{Mg}^{2+}, \mathrm{SO}_{4}^{2-}, \mathrm{CE}, \mathrm{HCO}_{3}^{-}, \mathrm{TZ}^{+}, \mathrm{TZ}^{-}, \mathrm{Na}^{+}, \mathrm{Cl}^{-}$ met en évidence une minéralisation des eaux liée au contact eau-roche (minéralisation temps de séjour). Enfin, le troisième qui prend en compte le $\mathrm{NO}_{3}{ }^{-}$montre une minéralisation anthropique issue des rejets urbains. En somme, les différents regroupements étudiés montrent que la minéralisation des eaux étudiées est liée à trois phénomènes importants. Il s'agit du phénomène de minéralisation par le contact eau-roche, du pluvio-lessivage, de la minéralisation issue des activités anthropiques. Cette méthode a été appliquée par plusieurs autres auteurs (Lallahem, 2002 ; Yao, 2009 ; Ahoussi et al., 2010 ; Babaye, 2012) et tous sont parvenus à la même conclusion.

La matrice de corrélation présentée dans le Tableau 4 avec un niveau de signification Alpha $=0,05$ ainsi que la Figure 8 montrent de bonnes corrélations entre certaines substances dissoutes prises deux à deux. C'est le cas des ions $\mathrm{Ca}^{2+}$ et $\mathrm{Mg}^{2+}, \mathrm{K}^{+}$et $\mathrm{Al}, \mathrm{Na}^{+}$et $\mathrm{Al}, \mathrm{Mg}^{2+}$ et $\mathrm{Al}$. Il existe également de très bonnes corrélations entre la somme des équivalents cations, la somme des équivalents anions (TZ) et certains éléments chimiques tels que l'aluminium (Al) et calcium $\left(\mathrm{Ca}^{2+}\right)$. Les mêmes corrélations s'observent entre la TDS et les cations majeurs $\left(\mathrm{Ca}^{2+}\right.$, $\mathrm{Mg}^{2+}, \mathrm{K}^{+}, \mathrm{Na}^{+}$), la TDS et les bicarbonates, la TDS et la silice. Ceci suggère que la TDS est constituée principalement par $\mathrm{Mg}^{2+}, \mathrm{Ca}^{2+}$, $\mathrm{HCO}_{3}{ }^{-}$et la silice. Il ressort de ces bonnes corrélations inter élémentaires que la plupart 
des éléments dissouts dans les eaux ont des origines communes.

Au cours de l'hydrolyse des minéraux primaires, les bicarbonates, les cations majeurs et la silice sont libérés suivant l'équation:

Minéraux primaires $+\mathrm{CO}_{2}+\mathrm{H}_{2} \mathrm{O} \rightarrow \mathrm{HCO}_{3}^{-}$ + minéraux secondaires + cations + silice.

Les eaux du bassin du Nchi sont majoritairement bicarbonatées calcimagnésiennes et en l'absence des roches carbonatées dans le bassin, tous les bicarbonates rencontrés sont d'origine atmosphérique $\left(\mathrm{CO}_{2}\right.$ atmosphérique et du sol). Ils se forment à partir des réactions chimiques suivantes :

$\mathrm{CO}_{2}+\mathrm{H}_{2} \mathrm{O} \rightarrow \mathrm{H}_{2} \mathrm{CO}_{3} ; \mathrm{H}_{2} \mathrm{CO}_{3} \rightarrow \mathrm{H}^{+}+\mathrm{HCO}_{3}^{-}$ (1)

Le $\mathrm{CO}_{2}$ induit l'hydrolyse des roches silicatées (Mazor, 2004):

$2 \mathrm{KAlSi}_{3} \mathrm{O}_{8}+6 \mathrm{H}_{2} \mathrm{O}+\mathrm{CO}_{2} \rightarrow \mathrm{Al}_{2} \mathrm{Si}_{2} \mathrm{O}_{5}$

$(\mathrm{OH})_{4}+4 \mathrm{SiO}(\mathrm{OH})_{2}+\mathrm{K}_{2} \mathrm{CO}_{3}$

$(\mathrm{Ca}, \mathrm{Fe}, \mathrm{Mg})\left(\mathrm{SiO}_{3}\right)+2 \mathrm{H}_{2} \mathrm{O}+2 \mathrm{CO}_{2}$

$(\mathrm{Ca}, \mathrm{Fe}, \mathrm{Mg})\left(\mathrm{HCO}_{3}\right)_{2}+\mathrm{SiO}(\mathrm{OH})_{2}$

$\mathrm{CaAl}_{2} \mathrm{Si}_{2} \mathrm{O}_{8}+2 \mathrm{CO}_{2}+3 \mathrm{H}_{2} \mathrm{O} \rightarrow \mathrm{Al}_{2} \mathrm{Si}_{2} \mathrm{O}_{5}(\mathrm{OH})_{4}$ $+2 \mathrm{HCO}_{3^{-}}+\mathrm{Ca}^{2+}$

$\mathrm{MgSiO}_{4}+2 \mathrm{CO}_{2}+\mathrm{H}_{2} \mathrm{O} \rightarrow \mathrm{Mg}^{2+} 2 \mathrm{HCO}_{3^{-}}+$ $\mathrm{SiO}_{2} \quad$ (5).

D'après le même auteur, les réactions (1) à (5) sont régies par un niveau de pression partielle de $\mathrm{CO}_{2}$ du sol relativement élevé. Ainsi, l'eau contenant le $\mathrm{HCO}_{3}^{-}$induit les interactions entre le $\mathrm{CO}_{2}$ et les roches. L'équilibrage des cations indique que les alcalino-terreux ( $\mathrm{Mg}$ et $\mathrm{Ca}$ ), qui constituent l'essentiel des cations de la zone d'étude, sont libérés au cours de la dissolution des minéraux primaires des roches, en particulier les feldspaths potassiques et/ou les minéraux ferromagnésiens (pyroxènes et olivines) par les relations de dissolution (2), (3) et (5). Ces éléments ont pratiquement le même comportement.

Les alcalins $\left(\mathrm{Na}^{+}\right.$et $\left.\mathrm{K}^{+}\right)$rencontrés dans les eaux du bassin versant du Nchi peuvent avoir plusieurs origines: l'altération des roches, le lessivage des sols, les apports atmosphériques par les précipitations, l'anthropisation, le piégeage des éléments dû à la morphologie du bassin.

Le sodium s'obtient par hydrolyse de l'albite en kaolinite suivant l'équation:

$$
\begin{gathered}
2 \mathrm{NaAlSi}_{3} \mathrm{O}_{8}+\underset{(\text { Albite) }}{2 \mathrm{CO}_{2}}+\underset{3 \mathrm{H}_{2} \mathrm{O}}{3} \rightarrow \\
\mathrm{Al}_{2} \mathrm{Si}_{2} \mathrm{O}_{5}(\mathrm{OH})_{4}+\underset{(\text { kaolinite })}{2 \mathrm{Na}^{+}+2 \mathrm{HCO}_{3}^{-}}+4 \mathrm{SiO}_{2}(6)
\end{gathered}
$$

Les principales sources d'ions nitrates dans les eaux sont : l'application d'engrais, les eaux usées, les décharges rejetées directement dans les eaux de surface (Yao, 2009), les eaux de ruissellement, le lessivage des terres par les précipitations et l'oxydation de l'azote. Elles proviennent également de l'oxydation des nitrites par les bactéries de la nitrification suite à l'infiltration des eaux usées. La concentration des nitrates en profondeur (eaux de nappes), indiquerait alors un système naturellement ouvert et par conséquent une infiltration verticale et directe vers la zone saturée (Yao, 2009).

Cependant, les faibles concentrations des nitrates observées dans le cours d'eau Nchi sont liées au processus de dénitrification. C'est en effet un processus bactérien de respiration alternatif. $\mathrm{Ce}$ phénomène biologique s'opère généralement, mais pas exclusivement dans le sol. Les bactéries dites dénitrifiantes favorisent la dénitrification en réduisant l'ion nitrate $\left(\mathrm{NO}_{3}{ }^{-}\right)$facilement soluble dans la solution du sol grâce aux liaisons hydrogènes qu'il établit avec les molécules d'eau.

L'aluminium et le fer sont des éléments assez abondants dans les roches cristallines anciennes soumises à une ferrallitisation intense dans la zone intertropicale (Lasm et al., 2008). Le fer et l'aluminium dans l'eau proviendraient principalement du lessivage des sols. Les plus fortes concentrations relevées en saison des pluies pourraient indiquer un apport par les eaux de ruissellement. En effet, le nettoyage de la surface des sols par des averses souvent violentes et généralisées après un démarrage de la saison des pluies, amène les plus fortes concentrations de matériaux en suspension, dont une partie est soluble. En surface, la densité des matériaux alumineux et ferrugineux est plus grande (latérite, cuirasse); il apparaît à cet effet logique d'attribuer à l'érosion des sols un rôle majeur dans la mise en solution de l'aluminium et du fer durant cette période de l'année. 
Dans la zone d'étude, la teneur en silice obtenue est supérieure à la somme des cations majeurs libérée lors de l'altération. Ceci correspondrait à l'altération des minéraux silicatés et à la solubilité de certains minéraux siliceux. Les ressources en eau du bassin versant du Nchi (faiblement minéralisées) ont une signature granito-gneissique et basaltique où la silice est l'élément le plus important.

\section{Paramètres environnementaux contrôlant le chimisme et la qualité des eaux du bassin versant du Nchi}

De ce qui précède, il en ressort que la qualité physico-chimique et bactériologique des eaux du bassin versant du Nchi sont contrôlés par plusieurs facteurs environnementaux; ce sont principalement la lithologie (nature des roches sous-jacentes, âge des roches, type et épaisseur de sols) et les activités anthropiques à travers les rejets urbains (Kamagaté, 2006 ; John et al., 2007 ; Ahoussi et al., 2010; Ahoussi et al., 2012 ; Ntep et al., 2014).

L'influence de la lithologie est liée au degré de solubilité de la roche (Kamagate et al., 2011; Xing, et al., 2013). Le bassin versant du Nchi est constitué de formations du socle (ensemble granito gneissique) et des basaltes de plateau déjà altérés. Ces ensembles rocheux sont d'âge ancien où tous les minéraux ont été lessivés en dehors du quartz, ce qui explique les faibles minéralisations des eaux de cette zone.

Les sols ferralitiques sont les plus représentés dans le bassin versant du Nchi. Ils sont constitués d'éléments tels que les argiles, les oxyhydroxides de fer et d'alumine (la kaolinite, la gibbsite l'hématite, la goethite) (Njoya, 2007). Ces sols ferralitiques, qui font l'essentiel de la couverture pédologique de la zone d'étude, sont généralement très épais et ralentissent de ce fait des infiltrations. Le substratum rocheux sous-jacent est alors isolé des eaux de percolation, réduisant ainsi le taux d'altération chimique et par conséquent la teneur en éléments chimiques.

L'effet de l'anthropisation sur les eaux souterraines du bassin versant du Nchi est indéniable. La dégradation des eaux souterraines mise en évidence par la présence des coliformes et streptocoques fécaux ainsi que des nitrates (Tita, 2008 ; Yao et al., 2012 ; Kouassi et al., 2012 ; Ntep et al., 2014) provient des déchets et autres polluants qui sont déversés dans la nature à travers les activités humaines (matières fécales des animaux à sang chaud). Ces déchets terminent dans la plupart des cas leur trajet dans les aquifères souterrains par infiltration verticale ou latérale. Cette contamination provient également des fosses d'aisance dont la majorité dans cette région sont à fond perdu. L'anthropisation est donc le facteur fondamental de la pollution chimique et bactériologique qui détériore la qualité des eaux de sources et de puits dans la zone d'étude où la démographie va grandissante.

L'eau constitue le transporteur privilégié d'un grand nombre de substances et d'organismes vers les nappes souterraines. Les eaux d'infiltration, se diffusant librement dans le sol et la zone saturée jusqu'au toit de la nappe, transportent sous forme dissoute ou par advection divers polluants. Une fois dans la nappe, le mouvement horizontal de l'eau transporte ces polluants organiques vers les sources d'approvisionnement en eau des populations. Les risques de pollution des eaux souterraines dépendent ainsi des caractéristiques des eaux d'infiltration (charges, débits) et des propriétés naturelles des couches géologiques qui séparent la nappe d'eau souterraine de la surface du sol (Andre et al., 2005; Xing et al., 2013; Yassir et Amira, 2013). En revanche, une fois la nappe contaminée par un polluant, elle le reste pendant longtemps. Ainsi, si les populations des centres urbains elles-mêmes sont responsables de la détérioration de la qualité des eaux des ouvrages complémentaires dans lesquels elles s'approvisionnent, il a été établi par Duchemin (1998) au regard des pollutions domestiques, une grille qui permet d'identifier le type d'usage possible pour valoriser au mieux les ressources disponibles tout en préservant au maximum la santé des populations. Les eaux des différents ouvrages ayant fait l'objet d'analyses bactériologiques dans le bassin versant du Nchi peuvent être destinées aux toilettes et baignades, au lavage des légumes, à la vaisselle, à la lessive et au lavage des sols. Pour la consommation, ces eaux demandent un traitement préalable dans 
le but de respecter les normes prescrites par l'OMS (Gilli et al., 2008) et dont l'extrait des teneurs limites des paramètres étudiés sont présentées dans le Tableau 5.

\section{Conclusion}

Les résultats de la présente étude ont permis de comprendre la signature minéralogique et bactériologique des eaux du bassin versant du Nchi. Ces résultats mettent en évidence sur le plan physico-chimique des eaux acides à neutres $(5.3 \leq \mathrm{pH} \leq 7,7)$ et faiblement minéralisées $[(15 \mu \mathrm{S} / \mathrm{cm} \leq$ conductivité électrique $\leq 164 \mu \mathrm{S} / \mathrm{cm})$ et $(184$ $\left.\left.\mu \mathrm{eq} / \mathrm{l} \leq \mathrm{TZ}^{+} \leq 1565 \mu \mathrm{eq} / \mathrm{l}\right)\right]$. L'ordre de grandeur décroissant des cations et anions majeurs dans les eaux du bassin versant du Nchi est le suivant: $\mathrm{HCO}_{3}^{-}>\mathrm{Ca}^{2+}>\mathrm{Mg}^{2+}$ $>\mathrm{Na}^{+}>\mathrm{K}^{+}>\mathrm{NO}_{3}{ }^{-} \geq \mathrm{Cl}^{-}$. Ce sont des eaux à faciès bicarbonatées calci-magnésiennes (hydrogéno-carbonaté-calcique et magnésienne). La restriction s'observe dans le puits $\mathrm{P}_{1}$, où on note une forte concentration des nitrates $(241,13 \mu \mathrm{eq} / \mathrm{l})$. Dans les eaux d'écoulement, la concentration moyenne des matières dissoutes totales pondérée par les débits est de 39,68 $\pm 11 \mathrm{mg} / \mathrm{l}$ avec de fortes concentrations en silice dissoute $(23,55 \pm 6,26$ $\mathrm{mg} / \mathrm{l})$ qui représente environ 59,33\%. Cette acidité et ces faibles minéralisations sont dues aux types de roches majoritairement granitogneissiques et basaltiques et à la grande épaisseur des sols ferrallitiques qui ralentit l'infiltration des eaux et l'altération des minéraux primaires. L'aluminium total est fortement représenté $(14,88 \mathrm{mg} / \mathrm{l})$ alors que le fer $(0,72 \mathrm{mg} / \mathrm{l})$ se trouve dans la même gamme de concentration que d'autres éléments majeurs dissouts.

Les variations spatiales montrent de fortes concentrations en éléments minéraux dissouts dans les eaux de nappe par rapport aux eaux d'écoulement. Celles-ci sont liées au temps de résidence beaucoup plus long des eaux de nappe dans le substratum rocheux et les sols.

Les variations saisonnières (teneurs en ions beaucoup plus élevées en saison sèche qu'en saison de pluies) ainsi que les différentes corrélations inter élémentaires montrent que l'origine principale des ions dans les eaux du cours d'eau Nchi est l'altération chimique par le processus de l'hydrolyse des minéraux primaires. Cette hydrolyse est rendue possible grâce à la présence du dioxyde de carbone atmosphérique et de celui qui est produit dans le sol par l'activité biologique à travers la respiration de la microfaune et de la microflore. Le sol étant ainsi la première interface entre l'eau et les minéraux. L'analyse bactériologique montre la présence des coliformes et des streptocoques fécaux, signe d'une dégradation de la qualité des ressources en eau liée à l'activité humaine et vectrice des maladies hydriques enregistrées actuellement dans cette zone. Ces ouvrages d'approvisionnement en eau dont les populations en sont largement tributaires nécessitent donc un suivi et des traitements adéquats (désinfection) avant leur consommation.

La lithologie ainsi que les activités anthropiques sont les principaux facteurs environnementaux qui contrôlent la qualité physico-chimique et bactériologique des eaux dans le bassin versant du Nchi. A ces principaux facteurs, s'ajoutent le climat, l'écoulement, le relief et la végétation.

\section{REFERENCES}

Abdou Babaye MS. 2012. Evaluation des ressources en eau souterraine dans le bassin de Dargol (Liptako-Niger). Thèse de Doctorat en cotutelle, Université de Liège Belgique et Université Abdou Moumouni Niger, 265 p.

Adrian H, Gallardo, Norio T. 2005. Hydrogeology and geochemical characterization of groundwater in a typical small-scale agricultural area of Japan. Journal of Asian Earth Sciences, 29: 18-28.

Ahoussi EK, Soro N, Koffi BY, Soro G, Biemi J. 2010. Origine de la minéralisation des eaux des aquifères discontinus sous couvert forestier de la zone Sud de la Côte d'Ivoire : cas de la région d'Abidjan-Agboville. Int. J. Biol. Chem. Sci., 4(3): 782-797.

Ahoussi KE, Koffi YB, Kouassi AM, Soro G, Soro N, Biemi J. 2012. Étude des caractéristiques chimiques et microbiologiques des ressources en eau 
du bassin versant du N'zi : cas de la commune de N'zianouan (Sud de la Côte d'Ivoire). Int. J. Biol. Chem. Sci., 6(4) : 1854-1873.

Andre L, Franceschi M, Pouchan P, Atteia O. 2005. Using geochemical data and modelling to enhance the understanding of groundwater flow in a regional deep aquifer, Aquitaine Basin, south-west of France. Journal of Hydrology, 305: 4062.

Bucrep. 2005. Rapport final du Troisième Recensement Général de la Population et de l'Habitat du Cameroun $3^{\text {ème }}$ (RGPH), $67 \mathrm{p}$.

Duchemin J. 1998. Grille de qualité des eaux d'alimentation humaine: paramètre et fréquence d'analyse des sources en fonction $\mathrm{du}$ substrat (alimentation et roches réservoirs) et de l'environnement. Document guide des classements des eaux de la DDAS, Loire Atlantique, 8 p.

Eblin SG, Soro GM, Sombo AP, Aka N, Kambiré O, Soro N. 2014. Hydrochimie des eaux souterraines de la région d'Adiaké (sud-est côtier de la Côte d'Ivoire). Larhyss Journal, 17: 193-214.

Gilli E, Mangan C, Mudry J. 2008. Hydrogéologie, Objets, Méthodes, Applications ( 2 édn). Dunod.

John DD, Subbba RN, Thirupathi BR, Srinivasa KVR, Subrahmanyam A. 2007. Hydrogeochimistry of the Sarada river basin, Visakhapatnam district, Andhra Pradesh. India. Environmental Geological, 52: 1331-1342.

Kamagate B, Gone DL, Doumouya I, Ouattara I, Ouedraogo M, Bamba A, Savane I. 2011. Relation nappe-rivière dans le bassin versant du Bandama en milieu de socle fissuré en Côte d'Ivoire : approche couplée hydrochimie-télédétection. Int. J. Biol. Chem. Sci., 5(1) : 206-216.

Kamagaté B. 2006. Fonctionnement hydrologique et origine des écoulements sur un bassin versant de milieu tropical de socle au Benin: bassin versant de la Donga (haute vallée de l'Ouémé). Thèse de Doctorat, Université de Montpellier II, Montpellier, $319 \mathrm{p}$.

Kouassi EA, Koffi YB, Kouassi AM, Soro G, Soro N, Biemi J. 2012. Étude des caractéristiques chimiques et microbiologiques des ressources en eau du bassin versant du N'zi : cas de la commune de N'zianouan (Sud de la Côte d'Ivoire). Int. J. Biol. Chem. Sci., 6(4): 1854-1873.

Lachassagne P, Wyns R. 2005. Aquifères de socle : nouveaux concepts, Application à la prospection et la gestion de la ressource en eau. Géosciences, 2: 32-37.

Lallahem S. 2002. Structure et modélisation hydrodynamique des eaux souterraines: Application à l'aquifère crayeux de la bordure nord du bassin de Paris. Thèse de Doctorat, Université des Sciences et Techniques de Lille, Lille, 219p.

Lasm T, Yao KT, Oga M-S, Kouamé F, Jourda JP, Kouadio E, Baka D. 2008. Analyse des caractéristiques physicochimiques des eaux souterraines en zone de socle protérozoïque de la région de Tiassalé (Sud de la Côte d'Ivoire). European Journal of Scientific Research, 20(3): 526-543.

Maréchal JC, Wins R, Lachassagne P, Subrahmanyam K, Touchard F. 2003. Anisotropie verticale de la perméabilité de l'horizon fissuré des aquifères de socle: concordance avec la structure géologique des profils d'altération. $C$. $R$. Géoscience, 335: 451-460.

Mazor E. 2004. Chemical and Isotopic Groundwater Hydrology. Marcel Drekker Inc. Publ: New York, 451 p.

Moore PJ, Jonathan B, Martin, Elizabeth J, Screaton. 2009. Geochemical and statistical evidence of recharge, mixing, and controls on spring discharge in an eogenetic karst aquifer. Journal of Hydrology, 376: 443-455.

Mouncherou OF, Moundi A, Ndam Ngoupayou JR, Andrew AA, Wandji P. 2011. Paramètres chimiques et source lithologique de la minéralisation des eaux souterraines des aquifères du Plateau Bamoun, Ligne Volcanique du Cameroun. Review of the Bulgarian Geological Society, 72 (3): 67-78.

Moundi A, Wandji P, Bardintzeff JM, Ménard JJ, Atouba Okomo LC, Mouncharou OF Reusser E, Bellon H, Tchoua F. 2007. Les basaltes éocènes à affinité 
transitionnelle du plateau Bamoun, témoins d'un réservoir mantellique enrichi sous la ligne volcanique du Cameroun. C. R. Géosciences, 339: 396406.

Moundi A. 2004. Les basaltes des plateaux du plateau Bamoun : Pétrologie-géochimie et Géochronologie : implications sur les sources de magmas, leurs contextes et évolutions géodynamiques. Thèse de Doctorat d'Etat, Université de Yaoundé I, Yaoundé, 392 p.

Njiné T, Monkiedje A, Nola M, et Sikati V. 2001. Evaluation de la charge polluante et de la charge bactérienne des rejets des stations à boue activées. Cahier Santé, 11: $79-84$.

Njoya A. 2007. Etude du gisement de kaolin de Mayouom (Ouest-Cameroun): Cartographie, minéralogie et géochimie. Thèse /PhD., Université de Yaoundé I, Yaoundé, 185 p.

Ntep F, Kengne IM, Ewodo Mboudou G, Nyochembeng N, Ekodeck GE. 2014. Influence of seasonal dynamics on groundwater resources quality in semitropical urban zone: case of the Biyémé upper stream catchment (Yaoundé, Cameroun). Int. J. Biol. Chem. Sci., 8(3): 1319-1335.

Omar R, Imane K, Fatiha K, Hamid C, Karima E, Abdalsalam I, Mostafa F, Youssef S, Khadija K, Driss B. 2014. Physico-Chemical Evaluation of Urban Wastewater of the Town of Sidi Kacem. Computational Water, Energy, and Environmental Engineering, 3: 30-35.

Rodier J. 2009. L'analyse de l'Eau; Eau Naturelle, Eau Résiduaire, Eau de Mer (9e édn). Dunod. Paris.

Savané I, Soro N. 2001. Caractérisation chimique et isotopique des eaux souterraines du Nord-Ouest de la Côte d'Ivoire: recharge des aquifères discontinus de la région d'Odienné. Africa Géoscience Review, 8(4): 379-390.

Tita MA. 2008. Water pollution of the Nkoup river system and its environmental impact on Foumbot town (Western Cameroon). Thèse de Doctorat/ph.D, Université de Yaoundé I, Yaoundé, 212 p.

Xiao J, Zhang-Dong J, Ding $\mathrm{H}$, Wang J, Zhang F. 2012. Geochemistry and solute sources of surface waters of the Tarim River Basin in the extreme arid region, NW Tibetan Plateau. J. Asian Earth Sci., 55: 162-173.

Xing L, Guo H, Zhan Y. 2013. Groundwater hydrochemical characteristics and processes along flow paths in the North China Plain. J. Asian Earth Sci., 70-71: 250-264.

Yao TK, Oga M-S, Fouché O, Baka D, Pernelle C, Biemi J. 2012. Évaluation de la potabilité chimique des eaux souterraines dans un bassin versant tropical : cas du Sud-Ouest de la Côte d'Ivoire . Int. J. Biol. Chem. Sci., 6: 70697086.

Yao TK. 2009. Hydrodynamisme dans les aquifères de socle cristallin et cristallophyllien du Sud-Ouest de la Côte d'Ivoire : cas du Département de Soubré. Apports de la télédétection, de la géomorphologie et de l'hydrogéochimie. Thèse de Doctorat en cotutelle, Conservatoire national des Arts et Métiers, France et Université de. Cocody, Côte d'Ivoire, 265p.

Yassir ME, Amira AA. 2013. Bacterial Contamination of Drinking Water in the Internally Displaced People Camps in South Darfur, Sudan Computational Water, Energy, and Environmental Engineering, 2: 10-12. 